\title{
The 12th Conference on the Formulation and Delivery of Bioactives
}

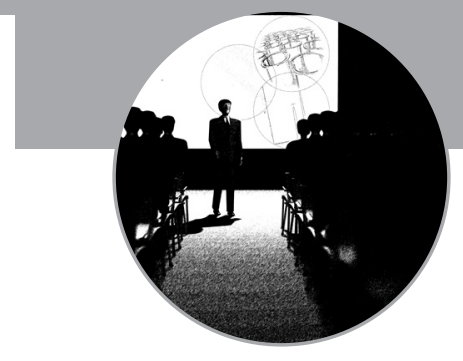

\section{Dunedin, New Zealand, 17-18 February 2010}

The effective delivery of bioactives (drugs) to the brain is a major challenge. Compared with the research intensity on the discovery of new drugs to treat neurological disorders, the effort on delivery of these therapies is limited. A second significant challenge is the delivery of large molecules, for example oligopeptides, proteins and oligonucleotides. These were the topics for discussion at the multidisciplinary 12 th Conference on the Formulation and Delivery of Bioactives.

Delivery of bioactives to the brain and the delivery of large molecules in general are two major challenges in therapeutic delivery. Researchers gathered at the 12 th Formulation and Delivery of Bioactives Conference in Dunedin, New Zealand, to present and discuss the problems and potential solutions to these challenges.

Formulating and delivering bioactives is a multidisciplinary challenge that engages two broad groups of people: those whose research focuses on formulating and delivery bioactives, and secondly those who need to delivery bioactives in the clinic or field, or for research purposes.

The term bioactive covers a wide range of compounds that can be variously categorized: chemical types (small organic molecules, peptides/proteins and oligonucleotides); therapeutic agents (drugs, biopharmaceuticals and vaccines); and nontherapeutic (poisons and toxins) agents, for various species (humans, companion animals, food-producing animals and wild animals - which may be pests), in different settings (clinic/hospital, home, farm and the wild). Given this diversity, it is not surprising that the Formulation and Delivery of Bioactives Conference (FDB) attracts a diverse group of people (pharmaceutical scientists, immunologists, chemists, physiologists, academics, research students, industrial and government researchers, and veterinary scientists).

For the last 12 years, the FDB has been held in February in Dunedin, New Zealand, the home of the most southerly university in the world (University of Otago, New Zealand). FDB is jointly sponsored by the FBD Research Theme of the University and the New Zealand Chapter of the Controlled Release Society. It attracts 100-120 participants who come to interact with keynote international and national scientists, and to build collaborative networks. A further important function of the conference is to give doctoral students opportunities not only to present their work, but to interact with leading international scientists and to learn how to effectively chair scientific sessions. It seems that questioning and discussion, following presentations at many international conferences these days, are what might euphemistically be described as 'limited'. This is a problem for our science and the small FDB conference tries to address this in a small way by giving doctoral students opportunity to co-chair sessions and stimulate discussion.

Each year the conference has keynote addresses on one or two major topics, in addition to oral and poster presentations, particularly from doctoral students, who compete for prizes to part-fund their attendance at the international CRS Conference, typically in the USA or Europe. Recent subjects have been: 'Imaging Techniques for Drug Delivery', 'Challenges in Oral Drug Delivery', 'Physicochemical Issues in Colloidal Drug and Vaccine Delivery' and 'Delivery of Bioactives to Companion Animals, Livestock and Wildlife'. This year the two foci of the conference were: 'Delivery of Bioactives to the Brain' and 'Challenges in the Delivery of Large Molecules'.

\section{Delivery of bioactives to the brain}

Given that most drugs are excluded from the brain and that funding spent on the discovery of neuropharmaceuticals far outweighs the spend on delivery to the brain, there is a clear need to promote this important research area.

Mark Habgood (University of Melbourne, Australia) opened the 'Delivery of Bioactives to the Brain' theme with an insightful address on 'Barriers to the Delivery of Drugs to the Injured Brain'. Habgood's research interests are

\section{Ian G Tucker}

School of Pharmacy, University of Otago, PO Box 56 Dunedin, New Zealand

Tel.: +64 34797296

Fax: +6434797034

E-mail: ian.tucker@

stonebow.otago.ac.nz 
in understanding the mechanisms of secondary tissue damage following spinal cord and traumatic brain injury, and, equally important, is his research on the function of active drug efflux mechanisms at the blood-CNS barrier interfaces during brain development. Although the first report of the restricted diffusion between blood and brain was made over 100 years ago, much remains to be understood about the blood-brain, blood-cerebrospinal fluid and cerebrospinal fluid-brain barriers, their tight junctions, influx and efflux transporters, and from Habgood's perspective, the time course of leakiness following trauma. Although clotting mechanisms seal damaged vessels, damage does create an opportunity for targeted delivery to the injured site as the barrier remains compromised for up to $6 \mathrm{~h}$ for $70-\mathrm{kDa}$ molecules and for up to 5 days for lower molecular weight compounds.

Methods of studying delivery of bioactives through the blood-brain barrier (BBB) was the focus of Joseph Nicolazzo's (Monash University, Melbourne, Australia) keynote address. Cellbased in vitro methods, and in situ and in vivo methods (e.g., carotid artery perfusion) together with knockout mouse models and specific inhibitors of transporters have been used by Nicolazzo to probe the BBB. Microdialysis, described by Hu Zhang (University of Otago), is another useful method to study the role of the BBB, given that it allows the measurement of free drug concentrations in the brain; however, sensitive assays are required given the low drug concentrations and small sample volumes.

After providing an overview of some of the strategies that have been used to enhance brain uptake (e.g., lipidization of bioactives, use of transporters and targeted drug-loaded colloidal systems), Ian Tucker (University of Otago) described some of their work on the use of bile salts to enhance the permeability of the BBB. The use of relatively high concentrations of bile salts as permeation enhancers is well known, but what is less clear is how these interesting amphiphilic molecules enhance cellular uptake of bioactives even at very low bile salt concentrations. Effects on membrane fluidity and transporter function appear to be involved.

Delivery to the brain from blood will not always be possible, in which case implants that release neuroactive substances for therapeutic benefit may be useful. Ruth Empson and Natalie Medlicott (both University of Otago) described work on a polymer implant for the sustained delivery of brain-derived neurotropic factor to the cerebellum. The cerebellum controls interactions with the environment, that is, the Purkinje neuron of the cerebellum provides an 'error correction' signal to bring a patient's intended movement in line with his/her actual movement; consequently, damage to the cerebellum may lead to ataxia. Empson and Medlicott have implanted polymer wafers on the surface of the cerebellum in mice and shown that release and diffusion of bioactive into the cerebellum is possible without adverse effects.

\section{Delivery of large molecules}

Many new drugs, either in the development pipeline or recently registered, are biopharmaceuticals. These medium- to high-molecular-weight molecules, particularly proteins, bring with them huge challenges in terms of their delivery and formulation, particularly with regards to their stabilization. Lene Jorgensen (Copenhagen) has studied protein instability due to adsorption, where the instability is caused by the unfolding of the adsorbed protein, and has investigated formulation approaches to stabilize the native ternary structure. Jorgensen studies unfolding in model systems (e.g., adsorption onto $200 \mathrm{~nm}$ Teflon ${ }^{\circledR}$ particles or adsorption at water-oil interfaces) using a range of instrumental techniques (e.g., differential scanning calorimetry, Fourier transform infrared spectroscopy and circular dichroism) and by microscopic observation. With such systems it is possible to study the influence of variables such as ionic strength, surfactants and sugars on adsorption and unfolding of the protein, and thereby design formulations that maintain native protein structure and activity during manufacture, shipping and long-term storage. However, the predictive theories are still unclear; her strong message was 'know your protein'.

Delivery (vis-à-vis formulation) of large molecules is the other major challenge. Thomas Rades (University of Otago) described novel colloidal delivery systems developed with his collaborators to improve the immunogenicity of poorly immunogenic antigens. These systems include: functionalized liposomes (e.g., mannosylated liposomes), cationic immune-stimulating complexes (Pluscoms), cubosomes and immunestimulating complex implants. Although some promising immune responses have been observed, it is apparent that relationships between the delivery modality and immunogenicity are still to be established. Gerrit Borchard (University of Geneva, Switzerland) stretched the audience's imagination giving a personal overview of the future of macromolecular drug delivery. For many years we have been familiar with the idea 
of designing drugs that mimic nature, so perhaps we need to be guided by nature in the design of truly novel delivery systems; for example, personalized medication systems comprising ink-jet chips coupled with microneedle arrays for injection on demand; or implanted mini-reactors able to deliver the drug when required.

As if the challenges for the delivery of macromolecules to humans were not enough, the delivery of such bioactives to wildlife has even greater obstacles to overcome. The brushtail possum (Trichosurus vulpecula), a native species of Australia, was progressively introduced into New Zealand over 100 years ago to establish a fur trade. It is now a pest destroying native flora as well as orchard crops, and is a reservoir for bovine TB. Various control strategies are being investigated. A fertility control approach using a gonadotropin-releasing hormone-toxin conjugate to target gonadotroph cells was described by Doug Eckery (Victoria University, New Zealand), but the issues of species-specific delivery and dosing in the wild are demanding. Arlene McDowell (University of Otago) described some potential formulation approaches to the challenges.

The conference was enriched by presentations by young scientists, some of which were on delivery of large molecules. For example,
Ilva Rupenthal (University of Auckland, New Zealand) described the efficacy of an in situ gelling system for the delivery of antisense oligonucleotides in a rat model of corneal damage, whereas Alexandra Kafka presented her PhD research on poly(alkylcyanoacrylate) nanoparticles for the peroral delivery of $\mathrm{D}$-Lys ${ }^{6}$-gonadotropin-releasing hormone. All abstracts of the conference can be found at http://bioactives. otago.ac.nz

\section{Looking ahead}

The next conference will be held in Dunedin in February 2011. It will again provide a multidisciplinary forum for discussion of cuttingedge issues in the formulation and delivery of bioactives.

Financial \& competing interests disclosure The author has no relevant affiliations or financial involvement with any organization or entity with a financial interest in or financial conflict with the subject matter or materials discussed in the manuscript. This includes employment, consultancies, honoraria, stock ownership or options, expert testimony, grants or patents received or pending, or royalties.

No writing assistance was utilized in the production of this manuscript. 